\title{
Engineering Majors' Cognitive Function Differentiation Ability
}

\section{Dr. Emre Tokgoz, Quinnipiac University}

Emre Tokgoz is currently the Director and an Assistant Professor of Industrial Engineering at Quinnipiac University. He completed a Ph.D. in Mathematics and another Ph.D. in Industrial and Systems Engineering at the University of Oklahoma. His pedagogical research interest includes technology and calculus education of STEM majors. He worked on several IRB approved pedagogical studies to observe undergraduate and graduate mathematics and engineering students' calculus and technology knowledge since 2011. His other research interests include nonlinear optimization, financial engineering, facility allocation problem, vehicle routing problem, solar energy systems, machine learning, system design, network analysis, inventory systems, and Riemannian geometry. 


\title{
Engineering Majors’ Mathematical Differential Function Conceptualization
}

\author{
Emre Tokgöz \\ Emre.Tokgoz@qu.edu \\ Industrial Engineering, School of Engineering, Quinnipiac University, Hamden, CT, 08518
}

\begin{abstract}
Derivatives of functions and their applications are important in Science-Technology-Engineering-Mathematics (STEM) fields. Pedagogical observations of the engineering students' conceptual knowledge of the derivative are necessary for finding an effective way of teaching derivative concept to students. Chain rule and its' applications in engineering education are particularly important noting that functions seen in real life engineering applications are complicated in nature and require applying the chain rule to find their derivatives. In this paper, undergraduate engineering students' conceptual derivative knowledge is observed qualitatively and quantitatively. Participants of this study were undergraduate engineering students enrolled at a mid-sized Northeast U.S. university. These students were asked to determine the derivative of a composition function as a part of a questionnaire consisting of calculus questions and were expected to apply the chain rule to solve the research question. Participants' cognitive approaches to solve the research question were investigated during the video recorded interviews. The research question was used as a part of another pedagogical research conducted at a large Midwest U.S. university to study undergraduate and graduate students' conceptual derivative knowledge. Quantitative results obtained in this article are bi-product of the participating students' schema classification introduced by Piaget and Garcia in 1989. ${ }^{15}$ The qualitative results are students' video recorded responses to the research question evaluated by using the concept image and concept definition of Vinner and Tall ${ }^{18}$.
\end{abstract}

Key Words: Function, derivative, chain rule, concept image and concept definition, schema classification, critical points.

\section{Introduction}

Derivatives of functions have a broad range of applications in engineering. It is particularly important for engineering students to have a good conceptual function derivative knowledge during their early undergraduate years due to its' frequent use in engineering majors. Weaknesses and strengths of engineering majors' conceptualization of function differentiation are studied by several researchers in recent years. ${ }^{20,22,23}$ These studies focused on engineering students ability to interrelate calculus concepts from various perspectives such as sketching the graph of a function by using asymptote, derivative, and limit knowledge as well as using trigonometric function and chain rule knowledge. ${ }^{22-25}$ The main theme of these studies is to observe the missing conceptual knowledge of Science-Technology-Engineering-Mathematics (STEM) students for solving ordinary calculus questions. 
Students' knowledge of functions' differentiation concept is studied by several researchers. ${ }^{2,10,13,21}$ It is observed by Orton (pp. 242) that students learn derivative as a rule and lack in the conceptual meaning of it during the applications for solving problems. ${ }^{13}$ Chain rule and its' applications in engineering education are particularly important noting that functions seen in real life engineering applications are complicated in nature and require applying the chain rule to find their derivatives. First year calculus students are observed to provide an example of what the chain rule is rather than explaining how it works. ${ }^{8}$ Intra, inter, and trans stages of triad classification is used for determining first year calculus students' conceptual chain rule; students are classified to be in the intra stage if they were able to apply the ordinary derivative and chain rules correctly but could not explain the relationship between them; students classified to be in the inter stage if they were able to apply all different derivative rules correct and recognize that they are related; participants are classified in the trans stage if they were able to construct and apply the chain rule correct. ${ }^{6}$ Students' conceptual ways of relating function composition and chain rule is investigated by Wangberg, Engelke, and Karakok after observing participants' use and interpretation of the chain rule while working on an online homework environment. ${ }^{20}$

The qualitative results of this study are determined by using the concept image and concept definition of Tall and Vinner. ${ }^{18}$ The concept image is described to be the total cognitive structure that is associated with the concept, which includes all the mental pictures and associated properties and processes; defined the concept definition to be a form of words used to specify that concept. Several studies reported misconceptions of students' encountering difficulties in changing their concept image; wrong images of the students conflicted with the chain rule definition. ${ }^{3,19}$ Students' image misconception of a given function and the misconception of the chain rule application on a given function is observed by Tokgöz and Gualpa ${ }^{25}$ similar to the findings of Vinner $^{19}$ and Aspinwall, Shaw and Presmeg². College students' difficulty in functions with cusp points or functions that are not polynomials are also observed by several researchers. ${ }^{4,17,25}$ Several participants' image misconception resulted in derivative application mistakes. ${ }^{21}$ Students were asked to sketch the graph of a function by solving limit, asymptote, and derivative related questions by Tokgöz et al. ${ }^{25}$; participants observed to have the most difficulty with the first derivative concept among the limit, function, asymptote, and derivative concepts.

In this article, the findings of a pedagogical research on engineering undergraduate students' knowledge of function derivatives will be presented. In the next section, the research methodology, research participants' background, and the research problem used for the data collection will be explained. Written (quantitative) and transcribed video recorded (qualitative) responses of the participants are displayed in the following section. Quantitative and qualitative data analysis is implemented with comparisons to the results in the literature at the end of this article. 


\section{Methodology, Participants \& Research Problem}

An Undergraduate Research Assistant (RA) collected the data evaluated in this work during a spring semester at a mid-sized North-Eastern University in the United States. The data collection procedure was approved by the Institutional Review Board (IRB) of the University. There were seventeen participants of this research majoring in one of the mechanical, industrial, or civil engineering programs and having completed the first three calculus courses of a four-year calculus course sequence. Each participant was given an hour to complete the questionnaire that consisted of 14 questions and the RA proctored the written questionnaire portion of the research. The questionnaire consisted of several calculus questions as well as questions that are designed to determine technology preferences of the participants to solve calculus questions. The author/researcher interviewed each participant approximately 30-40 minutes to further investigate the details of the written responses. The interviews are video recorded and transcribed to capture the information written by the participants during the interviews. Three out of seventeen participants did not participate to the video recorded interviews, however their written responses are determined to be eligible for the quantitative analysis but ineligible for the qualitative analysis. The participating students are compensated for both written and video recorded responses to the extent of their participation by using the IRB approved documentation. The derivative question related to the chain rule will be analyzed qualitatively and quantitatively in this article; the rest of the questionnaire questions will be analyzed elsewhere.

One of the objectives of this research is to gain an insight of engineering undergraduate students' ability to respond to a chain rule question for determining the improvement areas on engineering students' mathematics education. As a part of this objective, it is important to determine the weaknesses of the participants' chain rule and derivative knowledge as well as the extent to which participants concept image and concept definition coincide. The weaknesses observed in this article would pin-point the areas for improvement in engineering majors' mathematics education.

\section{Qualitative \& Quantitative Student Work}

In this section qualitative and quantitative results will be displayed based on the written and interview responses of the participants. The following quantitative results are the written responses of the participants during a spring school semester. The information written below in red are the written responses of the participants during the interviews. 
1. It $h(x)=\sin |x|$ a differentiable function for all real numbers in the not differentiable, please explain why,

The domain of this function is all real numbers. Therefon it is differentiable which means that you are able to differentiate it

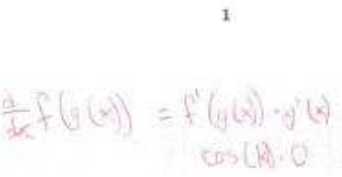

Response of Participant 1

1. Is $h(x)=\sin |x|$ a differentiable function for all real numbers in the domain? Please explain the domain of the function if it is differentiable. If it is not differentiable, please explain why.

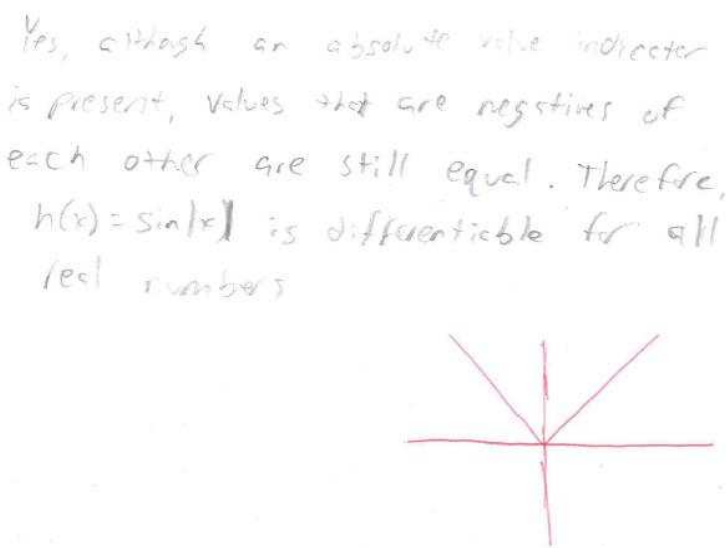

\section{Response of Participant 3}

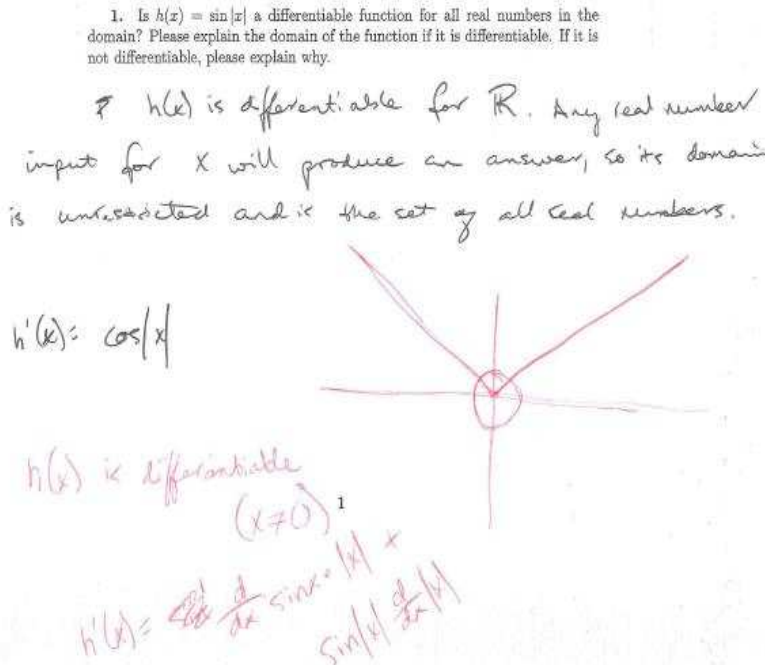

Response of Participant 5
1. Is $h(x)=\sin |x|$ a differentiable function for all real numbers in the domain? Please explain the domain of the function if it is differentiable. If it is not differentiable, please explain why.
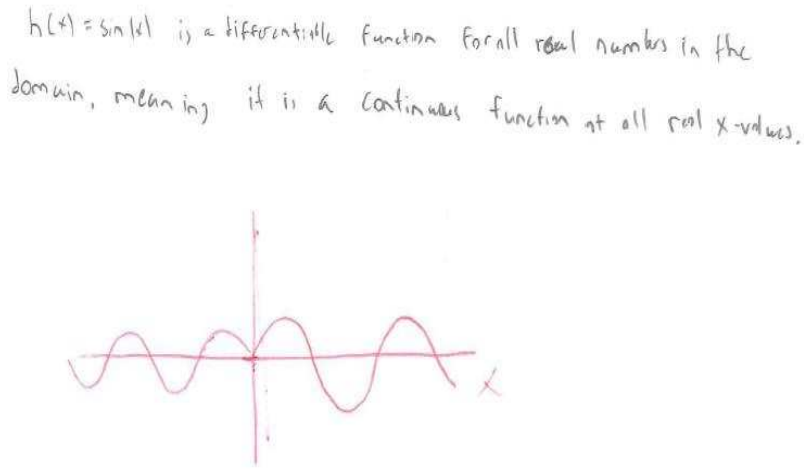

Response of Participant 2

1. Is $h(x)=\sin |x|$ a differentiable function for all real numbers in the domain? Please explain the domain of the function if it is differentiable. If it is not differentiable, please explain why

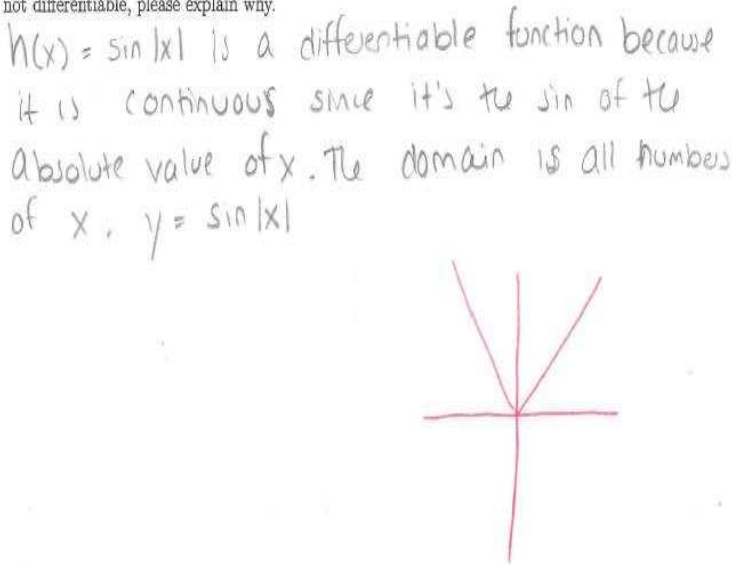

\section{Response of Participant 4}

1. Is $h(x)=\sin |x|$ a differentiable function for all real numbers in the domain? Please explain the domain of the function if it is differentiable. If it is not differentiable, please explain why

$h^{\prime}(x)=\cos (x)$
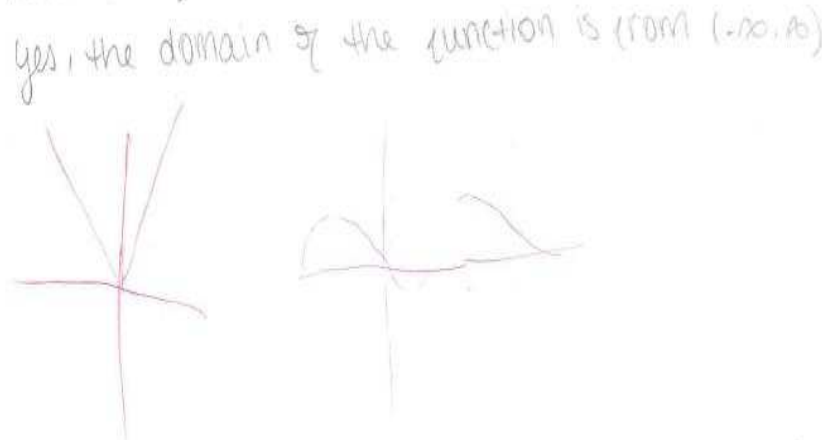
1. Is $h(x)=\sin |x|$ a differentiable function for all real numbers in the domain? Please explain the domain of the function if it is diferentiable. If it is$$
x(x)=2 \ln x
$$
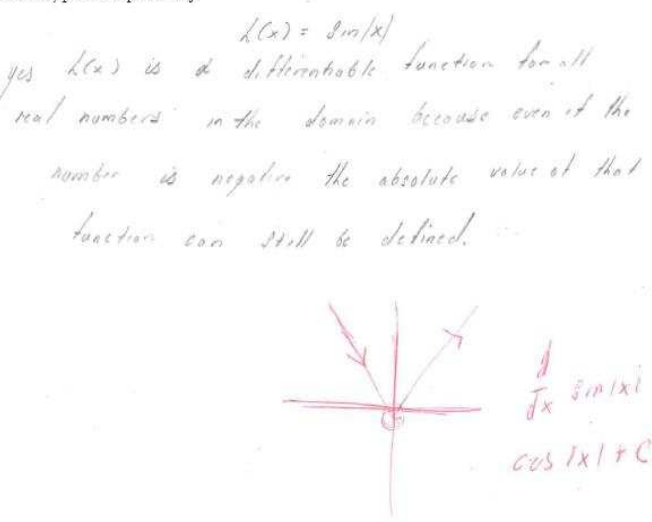

Response of Participant 7

1. Is $h(x)=\sin |x|$ a differentiable function for all real numbers in the domain? Please explain the domain of the function if it is differentiable. If it is not differentiable, please explain why.

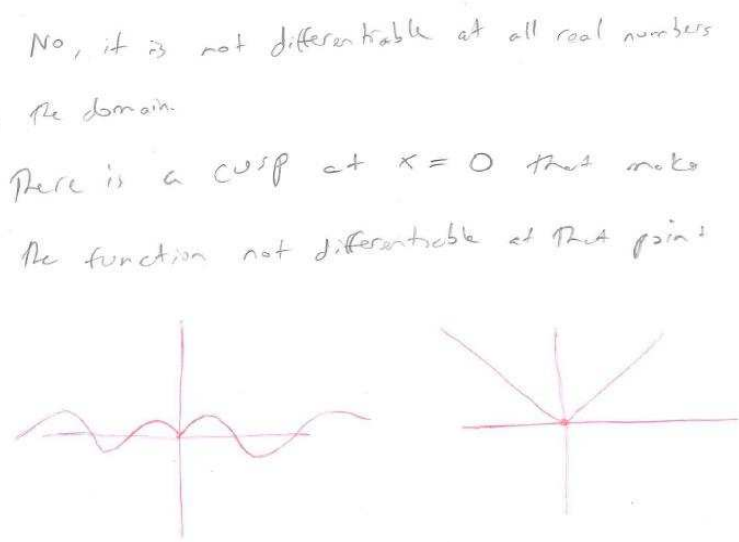

\section{Response of Participant 9}

1. Is $h(x)=\sin |x|$ a differentiable function for all real numbers in the domain? Please explain the domain of the function if it is differentiable. If it is not differentiable, please explain why.

$$
\text { yes; the fanction is differentiable for all real }
$$$$
\text { Mo,s in the domain, which is " } X \text { ". }
$$

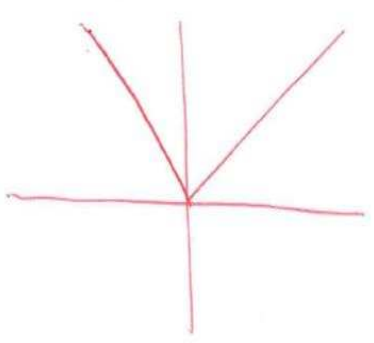

Response of Participant 11
1. Is $h(x)=\sin |x|$ a differentiable function for all real numbers in the domain? Please explain the domain of the function if it is differentiable. If it is not differentiable, please explain why

a domain is a list of all possible $x$-values

that allow the equation to exsist. With this

equation being a $\operatorname{SIN}$ FXN, $h(x)$ will be

between -1 and 1 . Although I de not remember

the exact meaning of differentiable, I am pretty

sure it is NCN differentiable because at $x=-\pi$ or $\pi$

the slope is vertically undefined.
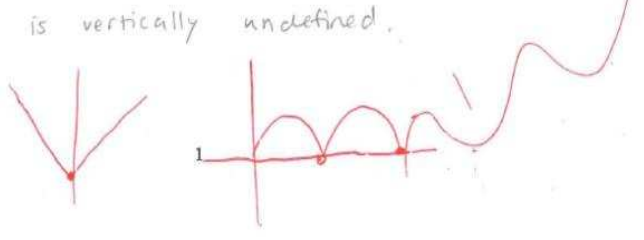

Response of Participant 8

1. Is $h(x)=\sin |x|$ a differentiable function for all real numbers in the domain? Please explain the domain of the function if it is differentiable. If it is not differentiable, please explain why. ?
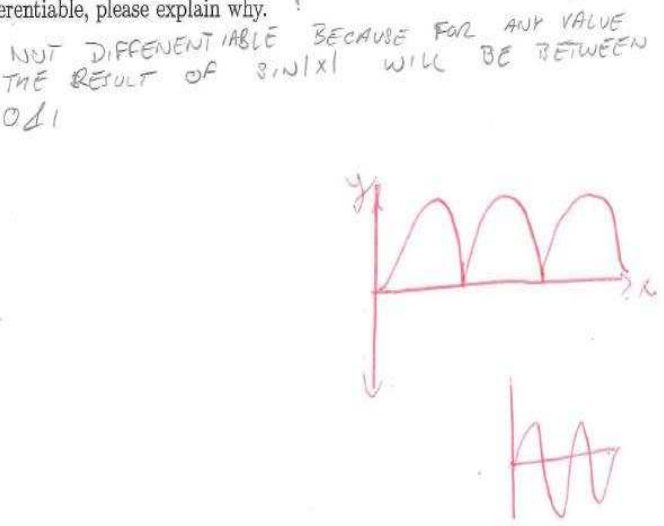

\section{Response of Participant 10}

1. Is $h(x)=\sin |x|$ a differentiable function for all real numbers in the domain? Please explain the domain of the function if it is differentiable. If it is not differentiable, please explain why.

I don't know what "differonticable function" is.

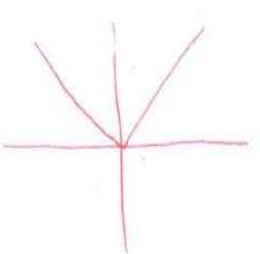

Response of Participant 12 


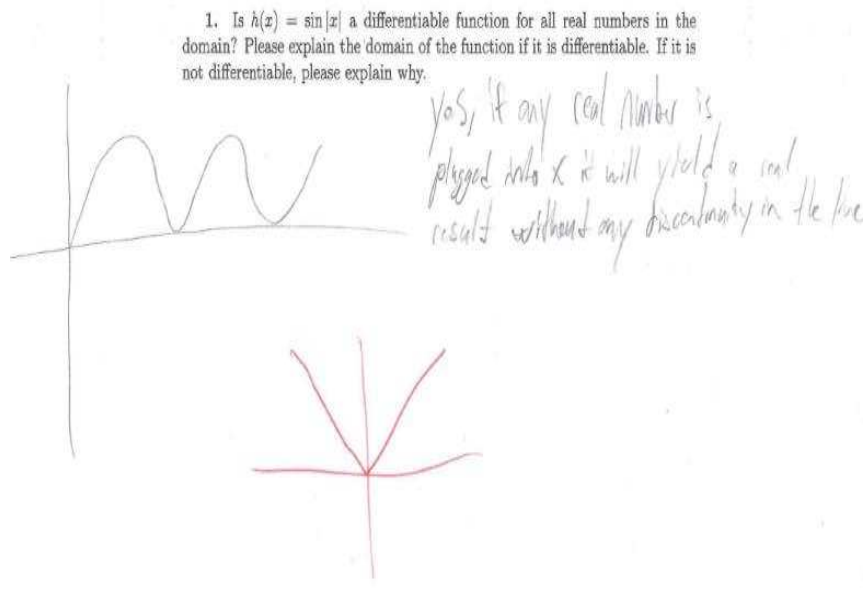

Response of Participant 13

1. I I f

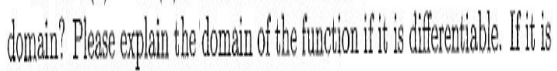

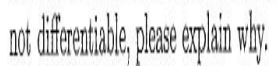

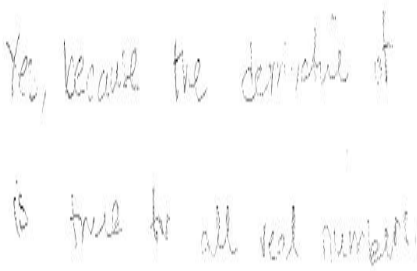

Response of Participant 15
1. Is $h(x)=\sin |x|$ a differentiable function for all real numbers in the ain the domain of the function if it is differentiable. If it is not differentiable, please explain why.

No since $x$ will always be positive

there is no way for the funcrion to

have a negative solution.

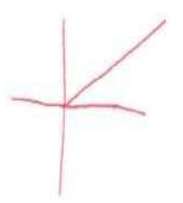

Response of Participant 14

1. Is $h(x)=\sin |x|$ a differentiable function for all real numbers in the domain? Please explain the domain of the function if it is differentiable. If it is not differentiable, please explain why.

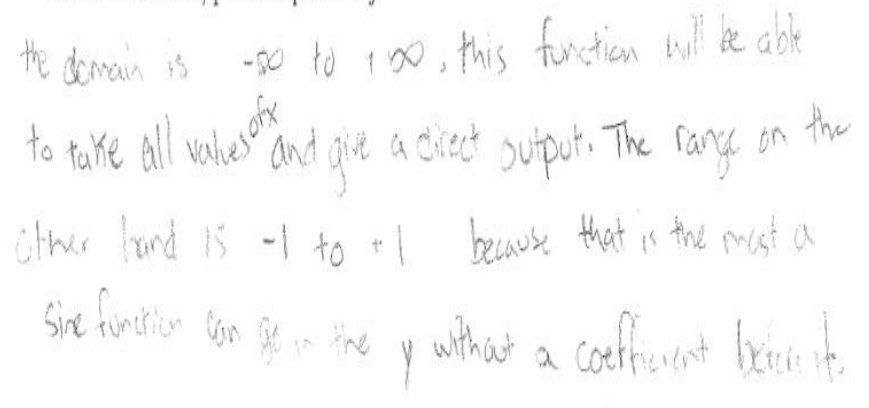

Response of Participant 16

1. Is $h(x)=\sin |x|$ a differentiable function for all real numbers in the domain? Please explain the domain of the function if it is differentiable. If it is not differentiable, please explain why.

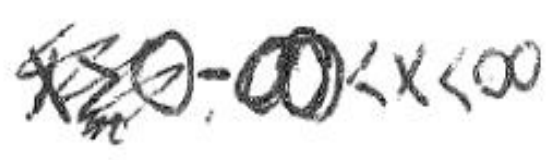

\section{itis a differentrabe enction forall neal numbers from-00 y to oo}

\section{Response of Participant 17}

Fourteen of the seventeen participants are interviewed by the author/researcher. These interviews are video recorded by the author and these responses are transcribed below. These transcriptions are not only important for understanding cognitive approach of students while responding to the question but also important for determining participants' alternative solution considerations used as a part of the qualitative data analysis. 
Participant 1: ...the first one is asking if $h(x)=\sin (|x|)$ is a differentiable function for all real numbers in the domain? So, I said it was because... you are able to differentiate it into other real numbers.

Interviewer: When you have $\sin (|x|)$, what makes it differentiable? What type of function is that? Is it a composition function or is it just a function itself? Is $\sin (\mathrm{x})$ differentiable?

Participant 1: Yes because... when you plug in $\sin (|x|)$ into the calculator you are getting something that never goes into negative values or has a vertical asymptote, so you can differentiate it to find a function.

Interviewer: Is the $|\mathrm{x}|$ a differentiable function?

Participant 1: Yes, except for one vertical asymptote.

Interviewer: Can you sketch the graph of $|\mathrm{x}|$ here?

Participant 1: (Sketches the graph on paper) ...it would be differentiable everywhere except for here (draws points on the graph)

Interviewer: ... Then can you also sketch the graph of $\sin (\mathrm{x})$ ?

Participant 1: (Sketches the graph of the function)

Interviewer: Okay. Is that all of it or...?

Participant 1: It would keep going. (Continues drawing on the graph)

Interviewer: Then, if you have $\sin (|x|)$, what type of function is that?

Participant 1: So $\sin (|x|)$ is anything on this side of the equation...

Interviewer: Okay. Can you sketch the graph of $\sin (|\mathrm{x}|)$ ?

Participant 1: (sketches the graph of $\sin (|x|)$ )...then it'd keep going

Interviewer: ... Then do you think $\sin (|\mathrm{x}|)$ is still differentiable for all values of $\mathrm{x}$ in the domain?

Participant 1: Except for here: when the differential of sine at $x=0$, the slope is zero.

Interviewer: ... when you look at the composition of those two, you end up with $\sin (|x|)$, right? Do you know any rules for composition functions' differentiability?

Participant 1: Not besides that they... when slope is zero it's not differentiable.

Interviewer: Do you know any differentiation rule when you have the composition of two functions?

Participant 1: ... if you have (draws $\mathrm{f}(\mathrm{g}(\mathrm{x}))$ ) ) and want to take the derivative of that it would be (writes the chain rule).

Interviewer: Okay, when you have this, can you tell me whether it's possible to find the derivative of $\sin (|\mathrm{x}|)$ ?

Participant 1: ... well the main place...

Interviewer: What is $\mathrm{g}(\mathrm{x})$ in this setting?

Participant 1: $\mathrm{G}(\mathrm{x})$ would be the $|\mathrm{x}|$...So differentiable of this would be...zero? So all of this would be zero.

Interviewer: So it can be differentiable where then?

Participant 1: Anywhere? Nowhere? I don't know.

Interviewer: After writing that, you had $\mathrm{g}^{\prime}(\mathrm{x})$ and you said $|\mathrm{x}|$ is not differentiable at zero.

Participant 1: You just end up with $\cos (|\mathrm{x}|)$ times zero. So, cosine is like (draws the graph of cosine) this you only have zero and then we have one so it would always be differentiable because that's...or no actually...that's like that, that's the top of an arc...So it's not? It is? I don't know.

Interviewer: Okay. So your final answer would be what for this? When you consider the chain rule and your prior answer to this. Do you think $\sin (|x|)$ is differentiable everywhere except the intersection points that you mentioned? Or is it still?

Participant 1: ...because you're multiplying by zero the whole time and zero is not differentiable because anything times zero is zero, I would say it is not differentiable.

...

Interviewer: ...Y You said this is a differentiable function for all real numbers...Is $|x|$ differentiable?

Participant 2: ...I wasn't sure so I made an estimation based off of what I thought of the sine wave to be with $|\mathrm{x}|$. So as $\mathrm{x}$, no matter what negative value it takes it makes it positive...ends up being a distorted sine wave.

Interviewer: Okay, can you sketch the graph of $|\mathrm{x}|$ for me?

Participant 2: ...so as $\mathrm{x}$ would change on the positive it's pretty easy. And on the negative...it would just be on the positive again. So, it would be that wave. So almost the inverse. I guess there is a point right there - the zero is not differentiable because it is not continuous.

Interviewer: ... is it not continuous?

Participant 2: Well, it's not that it's not continuous...I forget what it's called when it gets to a point and you can't 
get the tangent line on that point.

Interviewer: Okay...s so you're saying there's only one point where this function is not differentiable? Elsewhere it is differentiable?

Participant 2: Yes, that is what I am saying.

Interviewer: ...do you remember any rule that helps to figure out whether this function is differentiable or not?

Participant 2: No I do not.

Interviewer: What type of function $\sin (|\mathrm{x}|)$ is?

Participant 2: a trigonometric.

Interviewer: Is it a composition of two functions?

Participant 2: Yes.

Interviewer: Okay, is there any rule for us to figure out the differentiability of the function?

Participant 2: ...I don't remember it...I think there is chain rule?

Interviewer: ... So when you apply the chain rule, which one would cause a problem?

Participant 2: ... the absolute value.

Interviewer: Can you explain me your answer here? You're saying $\sin (|x|)$ is a differentiable function.

Participant 3: ... yeah sure, so I know that given the vertical line test there's one y value for every $x$ value and that sine of negative numbers...it's not gonna matter because sine wave is an oscillating function, so I said that it is still differentiable because the sine $(\mathrm{x})$ would be $\cos (\mathrm{x})$, right? Is that...? Yeah, differentiable - that would be the derivative.

Interviewer: Okay. Is $|\mathrm{x}|$ differentiable?

Participant 3: ...I thought so just because of the values, it wouldn't matter because a sine function is oscillating and the value that is the negative of the other is still the same

Interviewer: Can you sketch the graph of $|x|$ ?

Participant 3: Sure. (sketches the graph of $|x|$ )

Interviewer: So, is this differentiable everywhere? Can you find the derivative everywhere?...

Participant 3: ... I'm not sure it's been a while. I don't think so. Maybe if you analyzed it in two different parts. One from the positive $\mathrm{x}$ axis, one from the negative $\mathrm{x}$ axis.

Interviewer: Okay, so this is a differentiable function therefore $\sin (|x|)$ is differentiable?

Participant 3: Yes...And again, because of sine, the absolute value wouldn't matter because the values are still the same, so you can analyze it as a regular sine function.

Interviewer: ... You are saying the function is differentiable because it is continuous... Is $|\mathrm{x}|$ a differentiable function?

Participant 4: Yes, I believe so.

Interviewer: Can you sketch the graph of the function?

Participant 4: Okay (sketches the graph of $|x|$ )

Interviewer: Looking at the graph, do you think this function is differentiable?

Participant 4: Yes, because the function has no point where it breaks.

Interviewer: When you say "break" what do you mean?

Participant 4: There is no jump from one point to another or no asymptotes.

Interviewer: Is $\sin (\mathrm{x})$ differentiable?

Participant 4: Yes.

Interviewer: Okay. Is there a rule related to differential of composition of functions?

Participant 4: Not really.

Interviewer: Okay. Do you recall the chain rule?

Participant 4: Yeah!

Interviewer: If you apply the chain rule what would you get?

Participant 4: (Writes the derivative of $\mathrm{h}(\mathrm{x})$ based on the chain rule at the bottom of the page) I think this is it but I am not sure.

Interviewer: So that is what you will go with? 


\section{Participant 4: Sure!}

Interviewer: ...for the first question, you're saying the given function is differentiable. Can you explain your answer?

Participant 5: ...yes. Do you want me to say it or just write it down?

Interviewer: If you can just explain first. If you need to write, feel free.

Participant 5: ...first of all, I differentiated it just like a rule of thumb that...the differentiation of $\sin (\mathrm{x})$ is $\cos (\mathrm{x})$. And I just split it because those functions don't have any limitations... it's differentiable for all real numbers.

Interviewer: Okay. What's the graph of $|\mathrm{x}|$ ? Can you sketch that for me?

Participant 5: (sketches the graph.) $|\mathrm{x}|$ is like that?

Interviewer: Okay. Is there any place that this function is not differentiable?

Participant 5: Yeah, right at the little 'V.'

Interviewer: ... Then looking back at the original function, $\sin (|x|)$, would you change your answer, or would you still go with your answer? Your current answer.

Participant 5: ...I would change it because it's not differentiable at zero because there's no slope.

Interviewer: What would you change?

Participant 5: I'd say $h(x)$ is differentiable only for $x$ not equal to zero.

Interviewer: ... Do you know any rule for this function's differentiation?

Participant 5: ... Not off the top of my head, no.

Interviewer: Alright, if I tell you chain rule, would it ring a bell?

Participant 5: ... a little bit.

Interviewer: If you don't remember, it's OK.

Participant 5: That's where you do like the... you differentiate the inside times the outside, then do the differentiate of the outside and the inside

Interviewer: ... you write it as a formula? If you recall. If not, it's alright.

Participant 5: (writes the formula) I think it's something like that.

Interviewer: ...And then you find the derivative of $|\mathrm{x}|$.....and for any value that is not zero, you're saying this is differentiable?

Participant 5: Yes.

Interviewer: ... You're given $\sin (|x|)$, and you're saying "yes the domain of the function is from negative infinity to infinity." Do you remember the graph of $|\mathrm{x}|$ ?

Participant 6: It looks like... this.

Interviewer: ...is this a differentiable function?

Participant 6: Yes.

Interviewer: Okay....is it differentiable everywhere?

Participant 6: I think so...

Interviewer: ... Then is $\sin (|\mathrm{x}|)$ differentiable?

Participant 6: I thought so, I'm not sure.

Interviewer: $\mathrm{OK}$. What kind of function is $\sin (|\mathrm{x}|)$ ?

Participant 6: I thought $\sin (\mathrm{x})$ looked like this...but I wasn't sure about the sin absolute value of $\mathrm{x}$.

Interviewer: ... Is it a composition function?

Participant 6: I don't know.

Interviewer: ... you're given $\sin (|x|)$...can you explain your answer? Are you saying it's differentiable?

Participant 7: ...I said it is differentiable for all real numbers in the domain because it's an absolute value in the function that even if a negative number was entered in, then it would be allowed to operate as a function of sine. As long as the number was from, well, some divisible part of 0 to 360 .

Interviewer: Okay. Can you sketch the graph of $|x|$ for me please?

Participant 7: (sketches the graph of the function)

Interviewer: Is it differentiable everywhere?

Participant 7: I guess it wouldn't be here. 
Interviewer: ... if you have $\sin (|x|)$, and you want to find the derivative of it, do you know any rule for that?

Participant 7: ... I may have a little bit of difficulty explaining it, but I do know how to find the derivative of it.

Interviewer: Okay. Can you find it please for me?

Participant 7: The derivative of $\sin (\mathrm{x})$ would be $\cos (\mathrm{x})$. So, the derivative of $\sin (\mathrm{x})$ (writes the derivative) plus whatever constant would be applied to it.

Interviewer: ... would you need to find the derivative of $|x|$ at some point?

Participant 7: ... probably when it reaches a corner point such as probably when it lies at zero.

Interviewer: Can you please explain your answer?

Participant 8: Sure, when I looked at this I didn't exactly remember what was going on but I know that the domain is all the possible $\mathrm{x}$ values and differentiable is usually the derivative so I was thinking it will be along the lines where the slope is undefined; I know the sine wave is oscillating and at when $\mathrm{x}$ equals, wait hold on a moment, I know any $\mathrm{x}$ value would cause $\mathrm{h}(\mathrm{x})$ to be between -1 and 1 because it is like a circle, I am not really helping myself am I?

Interviewer: Is sine function differentiable?

Participant 8: It is a circle, no it is not because it is oscillating. When you take the derivative of sine it is cosine.

Interviewer: Does it mean differentiable?

Participant 8: Is it continuous? It is continuous. So sine is like (sketches a graph for the sine function). It is defined pretty much everywhere straight up and down. I am gonna say no

Interviewer: Is absolute value of $\mathrm{x}$ differentiable?

Participant 8: I know that in order it to be differentiable it needs to be continuous, it should not jump over things. Interviewer: Can you sketch the graph of $|\mathrm{x}| \ldots$ ?

Participant 8: Sketches the graph of $|x|$.

Interviewer: Is there any point that this function is not differentiable?

Participant 8: At exactly zero.

Interviewer: Going back to $\sin (\mathrm{x})$ is it differentiable?

Participant 8: (Sketches the graph of $\sin (|\mathrm{x}|)$ and points to the cusp points on the sketched graph and says at these points)... At these points non-differentiable

Interviewer: Alright, what are those points then?

Participant 8: Do you mean what $x$ values?

Interviewer: Yes

Participant 8: When $\mathrm{x}$ is pi or negative pi.

Interviewer: Are those the only points?

Participant 8: Yeah

Interviewer: ... What type of function is $\sin (|\mathrm{x}|)$ ?

Participant 8: Can't remember

Interviewer: Composition of functions sine and $|\mathrm{x}|$. Do you recall any rule related to composition of function differentiability?

Participant 8: No

Interviewer: Can you please explain me your answer?

Participant 9: The graph of the function appears to have a cusp at $x=0$ therefore if there is a cusp in the function then the function is not differentiable at that point.

Interviewer: Can you please sketch the graph of $|\mathrm{x}|$ please?

Participant 9: Sketches the graph of $|\mathrm{x}|$ and $\sin (|\mathrm{x}|)$

Interviewer: Where is $|\mathrm{x}|$ is not differentiable?

Participant 9: At 0 .

Interviewer: Is that the reason for $\sin (|x|)$ to be not differentiable?

Participant 9: It may not be but I thought it was because if you got this cusp here (pointing $\mathrm{x}=0$ on $|\mathrm{x}|$ ) and I see a cusp here (pointing $\mathrm{x}=0$ on $\sin (|\mathrm{x}|)$ ), that was my reasoning.

Interviewer: So $|x|$ is not differentiable at 0 therefore $\sin (|x|)$ is not differentiable at 0 ?

Participant 9: Yes 
Interviewer: Can you please explain your answer?

Participant 10: I just remember that it is all between 0 and 1 and I don't really remember what to do for $90 \%$ of that so I just said it is not differentiable.

Interviewer: Do you remember the graph of $|x|$ ?

Participant 10: I think so

Interviewer: Can you sketch it for me?

Participant 10: (Sketches a graph for $\sin (|x|))$ This is probably wrong.

Interviewer: What about the graph of $|\mathrm{x}|$ ?

Participant 10: (Sketches a graph of $\sin (\mathrm{x})$ ) This is the graph of $|\mathrm{x}|$. I got things totally massed up, I don't remember.

Interviewer: Can you please explain your answer?

Participant 11: I know the domain of the function. So it is asking if it is differentiable. I said it is differentiable for all real numbers in the domain because I understood $\mathrm{x}$ to be the domain and it represents the domain so I said it is differentiable for all real numbers.

Interviewer: Can you please sketch the graph of $|x|$ ?

Participant 11: Sketches the graph of $|x|$

Interviewer: Where is this function differentiable?

Participant 11: Differentiable for all real values on the $\mathrm{x}$-axis.

Interviewer: $\operatorname{So} \sin (|\mathrm{x}|)$ is differentiable for all $\mathrm{x}$ ?

Participant 11: Yes

Interviewer: Can you please explain your answer?

Participant 12: I didn't know differentiable means derivative but now I know.

Interviewer: Can you please sketch the graph of $|x|$ ?

Participant 12: Sketches the graph of $|x|$

Interviewer: Is this a differentiable function everywhere?

Participant 12: No.

Interviewer: Is there a place that this function doesn't have a derivative?

Participant 12: I don't know.

Interviewer: If it is not differentiable everywhere, $\operatorname{can} \sin (|\mathrm{x}|)$ be differentiable?

Participant 12: No

Interviewer: Why not?

Participant 12: From what I remember for absolute value, we don't have a derivative.

Interviewer: What do you mean?

Participant 12: If it is $\sin (\mathrm{x})$ then I know the derivative but I don't think I have ever seen $\sin (|\mathrm{x}|)$ before.

Interviewer: Even if it is the first time you are seeing it can you comment on whether it is differentiable or not?

Participant 12: Maybe I can find it. No, I don't think I can find the derivative of it.

Interviewer: So you are saying it is not differentiable everywhere.

Participant 12: No.

Interviewer: Can you explain the reason for that?

Participant 12: No

Interviewer: Can you please explain me your answer to the question?

Participant 13: I figured $h(x)=\sin (|x|)$ is a differentiable function for all values in the domain because if any real number plugged into $\mathrm{x}$ you would get real results, there would be no discontinuity

Interviewer: Do you recall the graph of $|\mathrm{x}|$ ? 
Participant 13: Yeah, kind of (sketches the graph of $|x|$ )

Interviewer: Is that differentiable everywhere?

Participant 13: Differentiable? Does that mean it has derivative?

Interviewer: Yeah

Participant 13: ...I think it should.

Interviewer: Is $\sin (\mathrm{x})$ differentiable? Can you find the derivative of $\sin (\mathrm{x})$ everywhere?

Participant 13: Yeah, it would be $\cos (\mathrm{x})$

Interviewer: Then $\sin (|\mathrm{x}|)$ becomes differentiable?

Participant 13: Yes

Interviewer: Can you please explain me your answer to the question?

Participant 14: Okay, since $|x|$ cannot be negative there is no way for the function to have a negative solution. So, if $\mathrm{h}(\mathrm{x})=\sin (|\mathrm{x}|)$ then no.

Interviewer: Can you sketch the graph of $|\mathrm{x}|$ ?

Participant 14: Sketches the graph of $|x|$ for $x>0$.

Interviewer: Is that the whole graph of $|\mathrm{x}|$ ?

Participant 14: I would say yes.

Interviewer: Is it differentiable everywhere or can you find the derivative everywhere?

Participant 14: I think you can find the derivative for the X-values but I am not sure.

Interviewer: Okay. For the function $\sin (|\mathrm{x}|)$, what $\mathrm{x}$-values would you not have the derivative?

Participant 14: I am pretty sure for negative $x$-values you cannot have derivative, so all has to be positive. So it has to be for all positive values less than 1 , I think.

Interviewer: Okay, can you also choose negative values?

Participant 14: You cannot choose negative values because there is absolute value, so it has to be positive.

Interviewer: So, for what $\mathrm{x}$-values this function won't be differentiable?

Participant 14: Anything greater than 1.

\section{Qualitative \& Quantitative Data Analysis}

In this section qualitative and quantitative data analysis of the collected data will be implemented. During the interviews the participants are asked to explain their written responses regardless of their correctness. Majority of the participants are asked to

- sketch the graph of $|x|$;

- determine the domain of differentiability of $|x|$;

- $\quad$ sketch the graph of $h(x)=\sin (|x|)$ (if they can);

- classify $h(x)=\sin (|x|)$ as a composition function and use the chain rule to determine the domain of differentiability of $h(x)=\sin (|x|)$.

Students' Participants' schema classification is determined according to the following knowledge-base by using a triad classification similar to the ones structured by Clark et al. ${ }^{6}$ and Tokgöz et al. ${ }^{25}$ : 
- Intra: Participants are classified in this group if they sketch the graph of $|\mathrm{x}|$ during the interviews.

- Inter: Participants who are classified to be in the intra level and are able to find the derivative of $h(x)=$ $\sin (|x|)$ during the interviews with some help are placed in this group.

- Trans: Participants who answered the research question correct without any help during the interviews are classified in this group.

The quantitative classification of the fourteen video recorded responses of the participants yield to the following schema classification:

\begin{tabular}{|c|c|c|}
\hline Intra & Inter & Trans \\
\hline 8 & 5 & 1 \\
\hline
\end{tabular}

The written responses indicated $93 \%$ of the participants' ability to sketch the graph of $|x|$ correct. $50 \%$ of the participants were not able to respond to the differentiability of $|\mathrm{x}|$ correct. $43 \%$ of the participants who were asked to sketch the graph of $\sin (|x|)$ during the interviews had the correct sketching. $\operatorname{Sin}(|x|)$ is determined to be differentiable by $76.5 \%$ of the students while $23.5 \%$ of the participants said $\sin (|x|)$ is not differentiable. Only six percent of the participants had the right reasoning for $\sin (|x|)$ to be not differentiable at a point. $66.7 \%$ of the participants were able to recall applicability of the chain rule to determine the derivative of $\sin (|x|)$ among those who were asked to determine the rule that needs to be applied to determine the derivative of the function and the rest of the participants were not able to recall the rule. The analysis of the participants' responses in this study indicated their difficulty of answering the derivative question by applying the chain rule on the contrary to the findings by Tokgöz et al. ${ }^{25}$ Function image misconception of students resulted in derivative application misconceptions similar to the findings in Tokgöz et al. ${ }^{25}$

The concept image is described to be the total cognitive structure that is associated with the concept, which includes all the mental pictures and associated properties and processes, and defined the concept definition to be a form of words used to specify that concept. ${ }^{18}$ In this work, participants' concept image and concept definition of derivative is investigated by using the corresponding definitions. In this study, participants were asked to sketch the graph of $|x|$ and determine whether this function is differentiable or not as a part of the interview questions. In addition, participants' ability to explain the differentiability of $\sin (|x|)$ was investigated both from concept definition and concept image perspectives. The concept definition and concept image knowledge of participants were well established for $|\mathrm{x}|$ while the contrary appeared for $\sin (|\mathrm{x}|)$. Transition from determining the differential of a basic function such as $|\mathrm{x}|$ to determining the differential of a complex function such as $\sin (|\mathrm{x}|)$ confused majority of the participants. Correct $\sin (|\mathrm{x}|)$ images drawn by some of the participants helped them to determine the correct answer to the differentiability of the function. Similarly, wrong $\sin (|x|)$ images drawn by the participants led them to wrong differentiability responses on these images. The chain rule is considered by hardly 
any participant for determining the differentiability domain of $\sin (|x|)$. The definition of $|x|$ is not used by any participant to determine the $\sin (|x|)$ in its differentiable function forms $\sin (x)$ when $x>0$ and $-\sin (x)$ when $x<0$. The complexity level of the composition function $\sin (|\mathrm{x}|)$ appeared to be the challenge for students for determining the differential of this function. Even though majority of the students had sufficient knowledge of $|\mathrm{x}|$, some of these students fail to use this knowledge to determine the derivative of $\sin (|x|)$.

\section{Conclusion}

In this work 17 engineering students' conceptual derivative knowledge based on a research question was investigated. An IRB (Institutional Review Board) approved research was implemented for collecting data during a spring semester at a mid-sized North-Eastern university in the United States. Qualitative and quantitative results were obtained from written and video recorded responses of the participating students.

Using the concept image and concept definition, the qualitative results indicated majority of the students' ability to match concept image and concept definition of $|\mathrm{x}|$ while the contrary was observed for the function $\sin (|\mathrm{x}|)$. Based on the written responses of the participants, the quantitative results indicated $93 \%$ of the participants' ability to sketch the graph of $|\mathrm{x}|$ correct. While $43 \%$ of the participants were able to sketch the graph of $\sin (|\mathrm{x}|)$ correct, $76.5 \%$ of the students said $\sin (|x|)$ is differentiable. $66.7 \%$ of the participants were able to recall applicability of the chain rule to determine the derivative of the function among the participants who were asked to determine the rule that needs to be applied to determine the derivative of $\sin (|x|)$. Only six percent of the participants had the right reasoning for $\sin (|\mathrm{x}|)$ to be non-differentiable at a point. Eight participants were classified in the intra stage if they faced difficulty while sketching the graph or determining the derivative of $|x|$; five participants were classified to be in the inter level if they were in the intra level and somehow faced difficulty in determining differentiability of $\mathrm{h}(\mathrm{x})=\sin (|\mathrm{x}|)$. Only one participant was qualified to be in the trans stage by answering the research question correctly.

In conclusion, it is observed that mathematics and engineering educators need to put more emphasis on concept image and concept definition relationship for students to have a better conceptual function and differentiation knowledge. The need for an efficient educational methodology appears to be compulsory to increase students' ability to use simple function knowledge (such as $|\mathrm{x}|$ ) as a part of a more complicated function (such as $\sin (|\mathrm{x}|)$ ). The use of technology can help students to determine cusp points by sketching the graphs of functions in the cases when they have insufficient conceptual knowledge.

\section{References}

1. Asiala, M., Brown, A., DeVries, D. J., Dubinsky, E., Mathews, D., \& Thomas K. (1996). A framework for research and curriculum development in undergraduate mathematics education. In J. Kaput, A. H. Schoenfeld, \& E. Dubinsky (Eds.), Research in collegiate mathematics education II (p/. 1-32). Providence, RI: American Mathematical Society and Washington, DC: Mathematical Association of America.

2. Asiala, M., Cottrill, J., Dubinsky, E., \& Schwingendorf, K. (1997). The Development of Students' Graphical Understanding of the Derivative. Journal of Mathematical Behavior, 16(4), 399-431. 
3. Aspinwall, L., Shaw, K. L., \& Presmeg, N. C. (1997). Uncontrollable mental imagery: Graphical connections between a function and its derivative, Educational studies in mathematics, 33, 301-317.

4. Baker, B., Cooley, L., \& Trigueros, M. (2000). A calculus graphing schema, Journal for Research in Mathematics Education, 31(5), 557-578.

5. Breidenbach, D., Dubinsky, E., Hawks, J., \& Nichols, D. (1992). Development of the process of function, Educational Studies in Mathematics, 23(3), 247-285.

6. Clark, J. M., Cordero, F., Cottrill, J., Czarnocha, B., DeVries, D. J., St. John, D., Tolias, G., \& Vidakovic, D. (1997). Constructing a schema: The case of the chain rule? Journal of Mathematical Behavior, 16(4), 345-364.

7. Cooley, L., Trigueros M., \& Baker B. (2007). Schema thematization: A theoretical framework and an example. Journal for Research in Mathematics Education, 38(4), 370 - 392.

8. Cottrill, J. (1999). Students' understanding of the concept of chain rule in first year calculus and the relation to their understanding of composition of functions. Doctoral Dissertation, Purdue University.

9. Dubinsky, E. \& McDonald M. A. (2002). APOS: A Constructivist Theory of Learning in Undergraduate Mathematics Education Research, The Teaching and Learning of Mathematics at University Level, 7 (3), 275-282.

10. Ferrini-Mundy, J., \& Gaudard, M. (1992). Secondary School Calculus: Preparation or Pitfall in the Study of College Calculus? Journal for Research in Mathematics Education, 23(1), 56-71.

11. Kashefi H., Ismail Z., \& Yusof Y. M. (2010). Obstacles in the Learning of Two-variable Functions through Mathematical Thinking Approach, International Conference on Mathematics Education Research, Social and Behavioral Sciences, 8, 173180.

12. Mathews, D., \& Clark, J. (1997). Successful students' conceptions of mean, standard deviation, and the Central Limit Theorem. Paper presented at the Midwest Conference on Teaching Statistics, Oshkosh, WI.

13. Orton, A. (1983). Students' Understanding of Differentiation. Educational Studies in Mathematics, 14, 235-250.

14. Parraguez, M. \& Ortac, A. (2010). Construction of the vector space concept from the viewpoint of APOS theory, Linear Algebra Appl. 432 (8), 2112-2124.

15. Piaget, J, \& Garcia, R. (1989). Psychogenesis and the history of science (H. Feider, Trans.). New York: Columbia University Press. (Original work published 1983).

16. Piaget, J., J.-B.Grize, A., Szeminska, \& V.Bang (1977). Epistemology and psychology of functions (J. Castellano`s and V.Anderson:Trans.)

17. Slavit, D. (1995). A growth-oriented route to the reification of function. In D. T. Owens, M. K. Reed, and G. M. Millsaps (Eds.), Proceedings of the seventeenth annual meeting of the North American Chapter of the international group for the psychology of mathematics education, 1, 284-290. Columbus, OH: ERIC Clearinghouse for Science, Mathematics, and Environmental Education.

18. Tall D. \& Vinner S. (1981). Concept Image and Concept Definition in Mathematics with particular reference to Limits and Continuity, Educational Studies in Mathematics, 12, 151-169.

19. Vinner, S. (1992). The function concept as a prototype for problems in mathematical learning. In E. Dubinsky \& G. Harel (Eds.), The concept of function: Aspects of epistemology and pedagogy (195-213). Washington, DC: Mathematical Association of America.

20. Wangberg, A., Engelke N., \& Karakok, G. (2011). Function composition and the chain rule in calculus. 14 ${ }^{\text {th }}$ SIGMAA Conference on Research in Undergraduate Mathematics Education, Preliminary report, Available at http://sigmaa.maa.org/rume/crume2011/RUME2011_FinalSchedule_files/PreliminaryReportsShortPapers/wangberg_proceed ings.pdf.

21. Zandieh, M. (2000). A Theoretical Framework for Analyzing Student Understanding of the Concept of Derivative. In E. Dubinsky, Schoenfeld, A., Kaput, J. (Ed.), Research in Collegiate Mathematics Education, IV (Vol. 8, pp. 103-127). Providence, RI: American Mathematical Society.

22. Tokgöz, E. (2015) Analysis of STEM Majors' Calculus Knowledge by Using APOS Theory on a Quotient Function Graphing Problem," ASEE Annual Meeting Proceedings, Mathematics Division, Seattle, Washington, Paper I.D. \#: 12664.

23. Tokgöz, E. (2012). Numerical Method/Analysis Students Derivative Concept Knowledge,” Int. J. New Trends in Educ. \& Their Implic., 3(4), pg. 125-134.

24. Tokgöz, E. (2016). Evaluation of Engineering \& Mathematics Majors' Riemann Integral Definition Knowledge by Using APOS Theory. ASEE Annual Conference Proceedings, paper ID\# 14461.

25. Tokgöz, E. and Gualpa, G. C. (2015). STEM Majors' Cognitive Calculus Ability to Sketch a Function Graph,” ASEE Annual Meeting Proceedings, Mathematics Division, Seattle, Washington, Paper I.D. \#: 12661. 